\title{
Alternativas terapéuticas farmacológicas para COVID-19
}

Arturo Pareja Cruz* 1,a ; Julio César Luque Espino 1,b

\section{RESUMEN}

En diciembre de 2019, China se convirtió en el centro de un brote de neumonía de causa desconocida que atrajo la atención mundial. Se trataba del nuevo coronavirus 2019 (2019-nCoV o SARS-CoV-2) que se ha expandido rápidamente. Hasta el 31 de marzo de 2020 se han reportado alrededor de 800000 casos y 38714 muertes en el mundo. En el Perú, se han informado 950 casos y 24 fallecidos. Se ha observado que el virus afecta con mayor frecuencia a adultos mayores, personas inmunocomprometidas o con comorbilidades. En esta revisión ofrecemos una vista panorámica de los conocimientos actuales relacionados con las alternativas terapéuticas para la COVID-19, desde fármacos con comprobada actividad antiviral que se han utilizado regularmente en otras enfermedades (arbidol, remdesivir, lopinavir/ritonavir, favipiravir) hasta medicamentos que usualmente han sido empleados como antiparasitarios (cloroquina e hidroxicloroquina) o antibacterianos (teicoplanina y azitromicina), pero que en la coyuntura actual retoman notoriedad por su posible y potencial eficacia frente a este virus. Asimismo, se resaltan los esquemas terapéuticos incluidos recientemente en la norma técnica emitida por el Ministerio de Salud en Perú (cloroquina, hidroxicloroquina e hidroxicloroquina/azitromicina). Si bien se han logrado avances importantes en la determinación de potenciales alternativas terapéuticas farmacológicas, es necesario realizar ensayos controlados aleatorizados para determinar la seguridad y la eficacia de medicamentos para el tratamiento de pacientes con COVID-19.

Palabras clave: Infecciones por coronavirus; Tratamiento; Terapia (Fuente: DeCS BIREME).

\section{Therapeutic alternatives for COVID-19}

\begin{abstract}
In December 2019, China became the center of a pneumonia outbreak of unknown cause that caught the world's attention. It was the new coronavirus 2019 (2019-nCoV or SARS-CoV-2), which has rapidly spread to the rest of the world. As of March 31,2020 , around 800,000 cases and 38,714 deaths have been reported worldwide. In Peru, 950 cases and 24 deaths have been reported. The virus has been found to affect most frequently elderly people, immunocompromised individuals or those with comorbidities. In this review article, we offer a comprehensive overview of current knowledge related to therapeutic alternatives for COVID-19, from drugs with proven antiviral activity that have been regularly used in other pathologies (arbidol, remdesivir, lopinavir/ritonavir, favipiravir) to drugs that have been usually used as antiparasitic (chloroquine and hydroxychloroquine) or antibacterial agents (teicoplanin and azithromycin), but nowadays have gained notoriety because of their possible and potential efficacy against COVID-19. Likewise, the therapeutic schemes recently included in the technical standard issued by the Ministry of Health of Peru (chloroquine, hydroxychloroquine and hydroxychloroquine/azithromycin) are highlighted. Although significant advances have been achieved in determining potential therapeutic alternatives, randomized controlled trials are needed to determine the safety and efficacy of medications for the treatment of patients with COVID-19.
\end{abstract}

Keywords: Coronavirus infections; Treatment; Therapy (Source: MeSH NLM).

1 Universidad de San Martín de Porres, Facultad de Medicina, Instituto de Investigación. Lima, Perú.

a Maestro en Salud Pública.

b Maestro en Investigación Clínica.

* Autor corresponsal 


\section{INTRODUCCIÓN}

Los coronavirus ( $\mathrm{CoV}$ ) son virus con ácido ribonucleico (ARN) monocatenario de sentido positivo, pertenecen a la familia Coronaviridae, subfamilia Orthocoronavirinae y pueden causar enfermedades en aves, mamíferos y humanos ${ }^{(1)}$.

En diciembre de 2019, la ciudad de Wuhan, provincia de Hubei, China, se convirtió en el centro de un brote de neumonía de causa desconocida y que atrajo una gran atención internacional. Para el 7 de enero de 2020, el agente causal de la enfermedad fue aislado y se determinó que se trataba de un nuevo coronavirus. El hecho se constituyó en el tercer brote de coronavirus reportado en el siglo XXI, el primero fue el SARS-CoV, en el 2002, que ocasiona el síndrome respiratorio agudo grave, y después el MERS-CoV, en el 2012, que provoca el síndrome respiratorio de Oriente Medio (2).

Este nuevo coronavirus 2019 (2019-nCoV o SARS-CoV-2) se ha venido expandiendo con rapidez, desde su origen en Wuhan, al resto del mundo. Hasta el 31 de marzo de 2020, se han reportado alrededor de 800000 casos de enfermedad por COVID-19 y 38714 muertes en el mundo. El país con la mayor cantidad de casos es Estados Unidos con 164610 y el que registra mayor número de muertes es Italia con 11591 decesos. En Perú se han reportado 950 casos y 24 muertes. Se ha observado que el virus afecta con mayor frecuencia a adultos mayores, personas inmunocomprometidas o con comorbilidades ${ }^{(3,4)}$.

En esta revisión ofrecemos una vista panorámica de los conocimientos actuales relacionados con el tratamiento y las alternativas terapéuticas para la COVID-19, desde fármacos que regularmente han sido utilizados en otras enfermedades con comprobada actividad antiviral, hasta medicamentos que usualmente se han empleado como antiparasitarios o antibacterianos pero que, en la coyuntura actual, retoman notoriedad por su posible y potencial eficacia en el tratamiento de la COVID-19. Dado que el conocimiento sobre este virus está evolucionando rápidamente, se insta a los lectores a mantenerse actualizados.

\section{ESTRATEGIA DE BÚSQUEDA}

La presente es una revisión narrativa sobre las alternativas terapéuticas farmacológicas para la infección por coronavirus SARS-CoV-2, COVID-19. Para ello, se realizó la búsqueda de información en las bases de datos Pubmed y Google Scholar. Las palabras que se utilizaron para realizar la búsqueda en dichas bases de datos fueron "nuevo coronavirus", "COVID-19", "terapia" y "tratamiento". Se seleccionaron los artículos que trataban sobre el tema publicados en inglés o español.

\section{GENERALIDADES SOBRE LA TERAPIA FARMACOLÓGICA}

El tratamiento de esta enfermedad es, esencialmente, de soporte y sintomático. La administración preventiva de antibióticos no debe realizarse sin una sobreinfección bacteriana confirmada microbiológicamente ${ }^{(5)}$, pero se requieren antibacterianos y antifúngicos si se prueba la presencia de bacterias u hongos. Hasta el momento, no existe un tratamiento aprobado para la COVID-19 ${ }^{(3)}$.

Todavía no existen vacunas autorizadas o agentes terapéuticos para tratar la infección por coronavirus, lo que pone de manifiesto la necesidad urgente de desarrollar vacunas eficaces o profilaxis posterior a la exposición para prevenir futuras epidemias. Varias características clínicas, genéticas y epidemiológicas de la COVID-19 se asemejan a la infección por SARS-CoV. Por lo tanto, los avances de la investigación sobre el tratamiento de este virus podrían ayudar a desarrollar agentes terapéuticos efectivos ${ }^{(1)}$.

Prajapat et al. realizaron una revisión sistemática para identificar las principales dianas terapéuticas farmacológicas en coronavirus. Encontraron siete elementos principales para los que se puede considerar el desarrollo de fármacos (proteína de pico, proteína de envoltura, proteína de membrana, proteasa, proteína de nucleocápside, hemaglutinina esterasa y helicasa). Además, otras 16 proteínas no estructurales también se pueden tomar en cuenta para el diseño de medicamentos ${ }^{(6)}$.

\section{ARBIDOL (UMIFENOVIR)}

Se ha demostrado que arbidol (nombre comercial de umifenovir) tiene, in vitro, un efecto antiviral directo en la replicación viral temprana del SARS-CoV (7). El arbidol (una pequeña molécula derivada de indol) inhibe la fusión, mediada por el virus, con la membrana objetivo, con el consecuente bloqueo de la entrada viral a las células blanco ${ }^{(8)}$. Este producto es utilizado como tratamiento para la influenza en Rusia y China, y se ha afirmado que fue efectivo en la terapia de COVID-19 en un rango de concentración de 10-30 $\mu \mathrm{M}$, in vitro. En China se ha iniciado un ensayo clínico aleatorizado controlado multicéntrico con arbidol en pacientes con COVID-19 ${ }^{(9)}$.

En el estudio de Wang et al., arbidol (umifenovir) mostró tendencia a mejorar la tasa de alta del paciente y a reducir la mortalidad en una pequeña cohorte de pacientes con COVID-19 ${ }^{(8)}$.

\section{REMDESIVIR (GS-5734)}

Remdesivir es un profármaco análogo de la adenosina. Según los datos recopilados, podría interferir en la polimerasa NSP12, in vitro ${ }^{(10)}$. Recientemente, ha sido reconocido como un antiviral prometedor contra una 
amplia gama de infecciones por virus ARN (incluidos SARS-CoV y MERS-COV) en células cultivadas, ratones y modelos primates no humanos. Además, se encuentra en desarrollo clínico para el tratamiento de la infección por el virus del Ébola ${ }^{(11)}$ y también para el SARS-CoV. En un reporte sobre el primer caso de COVID-19 en los Estados Unidos, el empleo de remdesivir resultó en una disminución de la carga viral en muestras nasofaríngeas y orofaríngeas, y la condición clínica del paciente mejoró ${ }^{(12,13)}$. A principios de febrero, se iniciaron dos ensayos clínicos de fase III para evaluar el uso de remdesivir por vía intravenosa en pacientes con COVID-19, que tienen como fecha de finalización estimada el mes de abril de $2020^{(14)}$.

\section{LOPINAVIR/ RITONAVIR}

Se conocía que los inhibidores de la proteasa lopinavir y ritonavir, utilizados para tratar la infección con el virus de la inmunodeficiencia humana $(\mathrm{VIH})$, podrían mejorar la condición de los pacientes con MERS-COV y SARS-CoV ${ }^{(10)}$.

La revisión sistemática de Yao et al. informa que la mayoría de los estudios in vitro han demostrado que lopinavir puede inhibir al SARS-CoV. Además, dos estudios de cohorte de pacientes con SARS-CoV revelaron que lopinavir/ritonavir desempeña un papel esencial en el resultado clínico, especialmente, en la etapa inicial. El tratamiento con lopinavir/ritonavir mejoró los resultados clínicos en algunos pacientes con MERS-CoV. Lopinavir podría ser un tratamiento efectivo para COVID-19 basado en la experiencia acumulada de los brotes de SARS y MERS ${ }^{(15)}$.

Lim et al. reportaron el caso de un paciente de 54 años con COVID-19 en Corea. Después de la administración de lopinavir/ritonavir, la carga viral disminuyó de manera significativa ${ }^{(16)}$.

En la India, Bhatnagar et al. publicaron un protocolo para el uso restringido de esta asociación en pacientes sintomáticos durante una emergencia de salud púbica. El protocolo fue aprobado por la autoridad sanitaria de dicho país y establece que los pacientes adultos hospitalizados por COVID-19 serán elegibles para recibir lopinavir/ritonavir durante 14 días después de firmar el consentimiento informado. Si este esquema es encontrado útil, se justifica una evaluación adicional mediante un diseño de ensayo de control aleatorio para el uso terapéutico de esta combinación en el futuro ${ }^{(17)}$.

\section{FAVIPIRAVIR (T-705)}

En China, el medicamento favipiravir se encuentra sometido a estudios clínicos para evaluar su eficacia y seguridad en el tratamiento de COVID-19 con resultados prometedores hasta ahora ${ }^{(18)}$. El favipiravir es un nuevo tipo de inhibidor de la ARN polimerasa dependiente de ARN (RdRp) ${ }^{(19)}$ que se convierte en una forma fosforribosilada activa (favipiravir-RTP) en las células y se reconoce como sustrato por la ARN polimerasa viral, y así inhibe la actividad de la ARN polimerasa ${ }^{(20)}$. El resultado preliminar de un ensayo clínico con 80 pacientes (incluidos el grupo experimental y el grupo control) indicó que favipiravir tuvo una acción antiviral más potente que la de lopinavir/ritonavir. No se observaron reacciones adversas significativas en el grupo de tratamiento con favipiravir que tuvo, significativamente, menos efectos adversos que el grupo de lopinavir/ritonavir. Por lo tanto, el favipiravir tendría una posible acción antiviral en COVID-19 ${ }^{(18)}$.

Tabla 1. Antivirales con posible y potencial eficacia frente a COVID-19

\begin{tabular}{|c|c|c|c|}
\hline Medicamento & Grupo & Evidencia in vitro & Evidencia clínica \\
\hline Arbidol (umifenovir) & $\begin{array}{l}\text { Inhibe la fusión } \\
\text { de la membrana }\end{array}$ & $\begin{array}{l}\text { Efecto antiviral en la } \\
\text { replicación viral in vitro } \\
\text { para SARS-CoV. }\end{array}$ & $\begin{array}{l}\text { - Se usa para la influenza en Rusia y China. } \\
\text { - Ensayo clínico aleatorizado controlado } \\
\text { multicéntrico en China para COVID-19. }\end{array}$ \\
\hline Remdesivir & $\begin{array}{l}\text { Profármaco análogo } \\
\text { de adenosina }\end{array}$ & $\begin{array}{l}\text { Fue efectivo en COVID-19 } \\
\text { en un rango de concentración } \\
\text { de } 10-30 \mu \mathrm{M} \text {, in vitro. }\end{array}$ & $\begin{array}{l}\text { - Estudios clínicos fase III en desarrollo } \\
\text { para COVID-19. } \\
\text { - Un reporte de caso en EE. UU. mostró } \\
\text { resultado favorable frente a COVID-19. }\end{array}$ \\
\hline Lopinavir/ritonavir & $\begin{array}{l}\text { Inhibidores de } \\
\text { la proteasa }\end{array}$ & $\begin{array}{l}\text { Podría interferir en la } \\
\text { polimerasa NSP12. }\end{array}$ & $\begin{array}{l}\text { - Utilizados para VIH. } \\
\text { - Estudios observacionales muestran resultados } \\
\text { favorables para SARS-CoV y MERS-CoV. } \\
\text { - Un reporte de caso en Corea mostró } \\
\text { resultado favorable frente a COVID-19. }\end{array}$ \\
\hline Favipiravir & $\begin{array}{l}\text { Inhibidor de la } \\
\text { ARN polimerasa }\end{array}$ & $\begin{array}{l}\text { Lopinavir puede inhibir } \\
\text { SARS-CoV. }\end{array}$ & $\begin{array}{l}\text { Estudios clínicos en desarrollo, } \\
\text { resultados preliminares favorables } \\
\text { para COVID-19. }\end{array}$ \\
\hline
\end{tabular}




\section{TEICOPLANINA}

La reutilización o replanteamiento del uso de medicamentos es una forma efectiva de identificar rápidamente fármacos con un perfil de seguridad conocido para tratar una enfermedad emergente. La teicoplanina es un glucopéptido utilizado para tratar infecciones bacterianas grampositivas (sobre todo, estafilocócicas) que se encontró activo, in vitro, contra el SARS-CoV. Se ha unido a la lista de moléculas que podrían usarse como arsenal terapéutico en COVID-19 ya que ha demostrado su eficacia contra varios virus como el ébola, virus de la gripe, flavivirus, virus de la hepatitis C, VIH, MERS-CoV y SARS-CoV (21).

En los coronavirus, la teicoplanina evita la liberación de ARN viral y, por lo tanto, la continuación del ciclo de replicación. Se ha observado que esta actividad se conserva en la COVID-19, por lo que la teicoplanina se coloca como un tratamiento potencial para pacientes con este virus ${ }^{(22,23)}$.

\section{CLOROQUINA}

La cloroquina, usada para tratar la malaria durante muchos años, tiene un gran potencial para el tratamiento de la COVID-19. Se investigan varios mecanismos posibles, por ejemplo, la cloroquina puede inhibir los pasos dependientes del $\mathrm{pH}$ de la replicación de varios virus con un potente efecto sobre la infección y propagación del SARS-CoV. Además, este fármaco tiene efectos inmunomoduladores, que suprimen la producción/liberación de TNF-a e IL-6. Varios estudios han encontrado que la cloroquina interfiere en la glucosilación de los receptores celulares de SARS $\mathrm{CoV}$ y funciona en las etapas de entrada y posentrada de la infección en las células Vero E6. Asimismo, se ha demostrado que la combinación de remdesivir y cloroquina inhibe eficazmente el virus in vitro ${ }^{(10)}$.

Publicaciones recientes apoyan la hipótesis de que la cloroquina puede mejorar el resultado clínico de pacientes infectados por SARS-CoV-2. Dado que hace unos días se descubrió que el virus utilizaba el mismo receptor de superficie celular que el SARS-CoV, la ECA2 (enzima convertidora de angiotensina 2) que se expresa en pulmón, corazón, riñón e intestino, se puede suponer que la cloroquina también interfiere en la glicosilación del receptor ECA2, y evita así la unión del virus a las células diana ${ }^{(24)}$. Wang y Cheng informaron que SARS-COV y MERSCoV aumentan la expresión de ECA2 en el tejido pulmonar, un proceso que podría acelerar su replicación y propagación (25).

La cloroquina también podría interferir en el procesamiento proteolítico de la proteína $M$ y alterar el ensamblaje y la gemación del virión. Finalmente, en la COVID-19, este fármaco podría actuar indirectamente reduciendo la producción de citoquinas proinflamatorias y/o activando las células T CD8+ antivirales ${ }^{(24)}$.

Cortegiani et al. plantean que existe una evidencia preclínica de efectividad y una evidencia de seguridad de uso clínico a largo plazo en otras enfermedades que justifican que se realice una investigación clínica sobre el uso de cloroquina en pacientes con COVID-19 (26).

\section{HIDROXICLOROQUINA}

La hidroxicloroquina difiere de la cloroquina por la presencia de un grupo hidroxilo. Ambos medicamentos son activos contra la malaria y tienen una farmacocinética similar, pero se diferencian en sus dosis tóxicas. La ventaja de la hidroxicloroquina es que puede usarse en dosis altas durante largos períodos con muy buena tolerancia ${ }^{(24,27)}$.

Existen más datos clínicos sobre la actividad anticoronavírica de la cloroquina que de la hidroxicloroquina. Ambos agentes son teóricamente similares en su actividad antiviral pero la cloroquina se asocia con mayores efectos adversos que la hidroxicloroquina y no está tan ampliamente disponible en algunos países. En pacientes con COVID-19, la cloroquina puede interactuar con lopinavir/ritonavir, lo que resulta en una prolongación del intervalo QT. Actualmente, se están investigando otros agentes terapéuticos para COVID-19 como los antivirales (oseltamivir, lopinavir/ritonavir o ribavirina), interferones e inmunoglobulinas intravenosas que no interfieren en la hidroxicloroquina ${ }^{(28)}$.

Zhou et al. proponen que la hidroxicloroquina, que muestra un efecto antiviral muy similar al de cloroquina, podría servir como un mejor enfoque terapéutico. Es probable que la hidroxicloroquina atenúe la progresión severa de COVID-19, inhibiendo la tormenta de citoquinas al suprimir la activación de las células T. Además, tiene un perfil clínico más seguro y es adecuada para las embarazadas ${ }^{(29)}$.

\section{HIDROXICLOROQUINA/AZITROMICINA}

Gautret et al. publicaron los resultados de un ensayo clínico no randomizado y de etiqueta abierta en el cual evaluaron el papel de la hidroxicloroquina en las cargas virales respiratorias.

Pacientes franceses con COVID-19 fueron incluidos en el estudio desde el inicio hasta el 16 de marzo. Diariamente, recibieron $600 \mathrm{mg}$ de hidroxicloroquina y también se analizaron sus cargas virales en hisopados nasofaríngeos. De acuerdo a la presentación clínica, se agregó azitromicina al tratamiento. Los pacientes no tratados de otro centro y los casos que no aceptaron la aplicación del protocolo se incluyeron como controles negativos.

La presencia o ausencia de virus en el día 6 después de la 
inclusión se consideró el punto final. Seis pacientes eran asintomáticos, 22 tenían síntomas de infección del tracto respiratorio superior y 8 tenían síntomas de infección del tracto respiratorio inferior. Veinte casos fueron tratados en este estudio y mostraron una reducción significativa de la carga viral en el día 6 después de la inclusión, en comparación con los controles. La azitromicina añadida a la hidroxicloroquina fue significativamente más eficiente para la eliminación del virus. Los autores concluyeron que, a pesar del pequeño tamaño de muestra, el estudio evidencia que el tratamiento con hidroxicloroquina se asocia significativamente con la reducción/desaparición de la carga viral en pacientes con COVID-19 y su efecto se ve reforzado por la azitromicina ${ }^{(30)}$.
Respecto a estas tres alternativas terapéuticas (cloroquina, hidroxicloroquina y la asociación de hidroxicloroquina/ azitromicina), el Ministerio de Salud de Perú, con fecha 29 de marzo de 2020, ha emitido la Resolución Ministerial 139-2020-MINSA que aprueba el Documento Técnico "Prevención y Atención de Personas Afectadas por COVID-19 en Perú". Este documento establece que, si bien no hay evidencia a partir de ensayos clínicos aleatorizados para recomendar tratamientos específicos, existen estudios internacionales con los fármacos en mención que ofrecen un limitado nivel de evidencia, por lo que se pone a consideración de los médicos especialistas tratantes, bajo su responsabilidad, los esquemas de tratamiento que las sociedades científicas nacionales sugieren ${ }^{(31)}$ (Tabla 2).

Tabla 2. Esquemas de tratamiento incluidos en el Documento Técnico "Prevención y Atención de Personas Afectadas por COVID-19 en Perú"

\begin{tabular}{llll}
\hline Medicamento & \multicolumn{1}{c}{ Dosis } & Duración & Vía de administración \\
\hline Cloroquina fosfato & $500 \mathrm{mg}$ cada 12 horas & $7-10$ días & Oral \\
Hidroxicloroquina & $200 \mathrm{mg}$ cada 8 horas & $7-10$ días & Oral \\
Hidroxicloroquina y & Hidroxicloroquina $(200 \mathrm{mg}$ & Hidroxicloroquina & Oral \\
azitromicina & cada 8 horas) y azitromicina & (7-10 días) y & \\
& $(500 \mathrm{mg}$ el primer día y & azitromicina (5 días) & \\
& $250 \mathrm{mg}$ cada 24 horas $)$ & &
\end{tabular}

\section{CONCLUSIONES}

Este nuevo brote de virus ha desafiado la infraestructura económica, médica y de salud pública no solo en el Perú sino en el mundo entero. Los esfuerzos actuales se centran en la contención y la cuarentena de las personas infectadas. Es evidente que el brote podría controlarse con una vacuna protectora para prevenir la infección por SARS-CoV-2, pero mientras no se disponga de ella es importante enfocarse también en las alternativas terapéuticas farmacológicas como las descritas en esta revisión. La investigación más profunda sobre la patogénesis de COVID-19 podría identificar objetivos terapéuticos apropiados para desarrollar antivirales específicos contra este patógeno. $\mathrm{Si}$ bien se han realizado avances importantes en la determinación de potenciales alternativas terapéuticas farmacológicas, se necesitan ensayos controlados aleatorizados para determinar la seguridad y la eficacia de los medicamentos para el tratamiento de pacientes con COVID-19. Mientras tanto, es importante seguir las recomendaciones y esquemas terapéuticos avalados por las autoridades sanitarias tanto a nivel mundial como nacional.

Contribuciones de los autores: Julio Luque participó en la búsqueda bibliográfica, organización de la información, redacción del artículo y conclusiones. Arturo Pareja participó en la concepción del artículo, la revisión de la bibliografía, organización de la información, redacción del artículo, revisión de la redacción y conclusiones.

Fuentes de financiamiento: Este artículo ha sido financiado por los autores.

Conflictos de interés: Los autores declaran no tener ningún conflicto de interés

\section{REFERENCIAS BIBLIOGRÁFICAS}

1. Shanmugaraj B, Siriwattananon K, Wangkanont K, Phoolcharoen W. Perspectives on monoclonal antibody therapy as potential therapeutic intervention for Coronavirus disease-19 (COVID-19). Asian Pac J Allergy Immunol. 2020; 38(1): 10-8.

2. Wang C, Horby PW, Hayden FG, Gao GF. A novel coronavirus outbreak of global health concern. Lancet Lond Engl. 2020; 395(10223): 470-3.

3. Singhal T. A review of Coronavirus Disease-2019 (COVID-19). Indian J Pediatr. 2020; 87(4): 281-6.

4. Johns Hopkins University-Medicine. Coronavirus Resource Center [internet]; 2020. Disponible en: https://coronavirus.jhu.edu/ map.html

5. Wujtewicz M, Dylczyk-Sommer A, Aszkiełowicz A, Zdanowski S, 
Piwowarczyk S, Owczuk R. COVID-19- what should anaethesiologists and intensivists know about it?. Anaesthesiol Intensive Ther. 2020; 52(1): 34-41.

6. Prajapat M, Sarma P, Shekhar N, Avti P, Sinha S, Kaur H, et al. Drug targets for corona virus: a systematic review. Indian J Pharmacol. 2020; 52(1): 56-65.

7. Deng L, Li C, Zeng Q, Liu X, Li X, Zhang H, et al. Arbidol combined with $\mathrm{LPV} / \mathrm{r}$ versus LPV/r alone against Corona Virus Disease 2019: a retrospective cohort study. J Infect. 2020.

8. Wang Z, Yang B, Li Q, Wen L, Zhang R. Clinical features of 69 cases with Coronavirus Disease 2019 in Wuhan, China. Clin Infect Dis. 2020.

9. Wang Z, Chen X, Lu Y, Chen F, Zhang W. Clinical characteristics and therapeutic procedure for four cases with 2019 novel coronavirus pneumonia receiving combined Chinese and Western medicine treatment. Biosci Trends. 2020; 14(1): 64-8.

10. Guo Y-R, Cao Q-D, Hong Z-S, Tan Y-Y, Chen S-D, Jin H-J, et al. The origin, transmission and clinical therapies on coronavirus disease 2019 (COVID-19) outbreak: an update on the status. Mil Med Res. 2020; 7(1): 11.

11. Wang $M$, Cao R, Zhang L, Yang X, Liu J, Xu M, et al. Remdesivir and chloroquine effectively inhibit the recently emerged novel coronavirus (2019-nCoV) in vitro. Cell Res. 2020; 30(3): 269-71.

12. Wu Y-C, Chen C-S, Chan Y-J. The outbreak of COVID-19: an overview. J Chin Med Assoc. 2020; 83(3): 217-20.

13. Holshue ML, De Bolt C, Lindquist S, Lofy KH, Wiesman J, Bruce $H$, et al. First case of 2019 Novel Coronavirus in the United States. N Engl J Med. 2020; 382(10): 929-36.

14. Li G, De Clercq E. Therapeutic options for the 2019 novel coronavirus (2019-nCoV). Nat Rev Drug Discov. 2020; 19(3): 14950.

15. Yao T-T, Qian J-D, Zhu W-Y, Wang Y, Wang G-Q. A systematic review of lopinavir therapy for SARS coronavirus and MERS coronavirus: a possible reference for coronavirus disease-19 treatment option. J Med Virol. 2020.

16. Lim J, Jeon S, Shin HY, Kim MJ, Seong YM, Lee WJ, et al. Case of the index patient who caused tertiary transmission of COVID-19 infection in Korea: the application of Lopinavir/Ritonavir for the treatment of COVID-19 infected Pneumonia monitored by quantitative RT-PCR. J Korean Med Sci. 2020; 35(6): e79.

17. Bhatnagar T, Murhekar MV, Soneja M, Gupta N, Giri S, Wig N, et al. Lopinavir/ritonavir combination therapy amongst symptomatic coronavirus disease 2019 patients in India: protocol for restricted public health emergency use. Indian J Med Res. 2020.

18. Dong L, Hu S, Gao J. Discovering drugs to treat coronavirus disease 2019 (COVID-19). Drug Discov Ther. 2020; 14(1): 58-60.

19. Delang L, Abdelnabi R, Neyts J. Favipiravir as a potential countermeasure against neglected and emerging RNA viruses. Antiviral Res. 2018; 153: 85-94.

20. Furuta $\mathrm{Y}$, Komeno T, Nakamura T. Favipiravir (T-705), a broad spectrum inhibitor of viral RNA polymerase. Proc Jpn Acad Ser B Phys Biol Sci. 2017; 93(7): 449-63.

21. Baron SA, Devaux C, Colson P, Raoult D, Rolain J-M. Teicoplanin: an alternative drug for the treatment of coronavirus COVID-19?. Int J Antimicrob Agents. 2020; 105944

22. Zhou N, Pan T, Zhang J, Li Q, Zhang X, Bai C, et al. Glycopeptide antibiotics potently inhibit Cathepsin $\mathrm{L}$ in the late endosome/ lysosome and block the entry of Ebola Virus, Middle East Respiratory Syndrome Coronavirus (MERS-CoV), and Severe Acute Respiratory Syndrome Coronavirus (SARS-CoV). J Biol Chem. 2016; 291(17): 9218-32.

23. Zhang J, Ma X, Yu F, Liu J, Zou F, Pan T, et al. Teicoplanin potently blocks the cell entry of 2019-nCoV. BioRxiv. 2020.

24. Devaux CA, Rolain J-M, Colson P, Raoult D. New insights on the antiviral effects of chloroquine against coronavirus: what to expect for COVID-19?. Int J Antimicrob Agents. 2020; 105938.

25. Wang $P$, Cheng Y. Increasing host cellular Receptor-AngiotensinConverting Enzyme 2 (ACE2) expression by Coronavirus may facilitate 2019-nCoV Infection. BioRxiv. 2020.

26. Cortegiani A, Ingoglia G, Ippolito M, Giarratano A, Einav S. A systematic review on the efficacy and safety of chloroquine for the treatment of COVID-19. J Crit Care. 2020.

27. Wellems TE, Plowe CV. Chloroquine-resistant malaria. J Infect Dis. 2001; 184(6): 770-6.

28. Sahraei Z, Shabani M, Shokouhi S, Saffaei A. Aminoquinolines against Coronavirus Disease 2019 (COVID-19): chloroquine or hydroxychloroquine. Int J Antimicrob Agents. 2020; 105945.

29. Zhou D, Dai S-M, Tong Q. COVID-19: a recommendation to examine the effect of hydroxychloroquine in preventing infection and progression. J Antimicrob Chemother. 2020.

30. Gautret P, Lagier J-C, Parola P, Hoang VT, Meddeb L, Mailhe M, et al. Hydroxychloroquine and azithromycin as a treatment of COVID-19: results of an open-label non-randomized clinical trial. Int J Antimicrob Agents. 2020; 105949.

31. Resolución Ministerial $\mathrm{N}^{\circ} 139-2020$-MINSA [internet]. Documento Técnico: Prevención y atención de personas afectadas por COVID-19 en el Perú. Disponible en: https://www.gob.pe/ institucion/minsa/normas-legales/465962-139-2020-minsa

\section{Correspondencia:}

Arturo Pareja Cruz

Dirección: Av. Alameda del Corregidor 1531, Urb. Los Sirius, La Molina. Lima, Perú.

Teléfono: 3652300

Correo electrónico: aparejac@usmp.pe

Recibido: 30 de marzo de 2020.

Evaluado: 02 de abril de 2020 .

Aprobado: 10 de abril de 2020 .

(c) La revista. Publicado por Universidad de San Martín de Porres, Perú.

(c) Br Licencia de Creative Commons Artículo en acceso abierto bajo términos de Licencia Creative Commons Atribución 4.0 Internacional. (http://creativecommons.org/licenses/by/4.0/)

ORCID iDs

Arturo Pareja Cruz C https://orcid.org/0000-0002-5988-5515

Julio César Luque Espino https://orcid.org/0000-0001-8868-2883 